TRIBUNAL EUROPEO DE DERECHOS HUMANOS 



\title{
LOS ESTÁNDARES DE DERECHO ELECTORAL A LA LUZ DE LA JURISPRUDENCIA DEL TRIBUNAL EUROPEO DE DERECHOS HUMANOS Y DEL CÓDIGO DE BUENAS PRÁCTICAS DE LA COMISIÓN DE VENECIA
}

\author{
AMAYA ÚBEDA DE TORRES \\ Letrada del Consejo de Europa
}

TRC, núm. 46, 2020, pp. 563-584

ISSN 1139-5583

\begin{abstract}
SUMARIO
I. Introducción. II. La protección de los derechos políticos en el Convenio Europeo de Derehos Humanos: análisis de los principios en materia electoral a la luz de la jurisprudencia. II. La protección de la democracia a través de los estándares de soft law: la contribución de la Comisión de Venecia. IV. Reflexiones finales.
\end{abstract}

\section{INTRODUCCIÓN}

La celebración de elecciones, como proceso, implica un conjunto de elementos y, sobre todo, el paso por una serie de etapas que forman parte del llamado ciclo electoral y que implican el ejercicio de los derechos de participación política. Si bien el período electoral como tal exige que se garanticen todos los aspectos necesarios para poder asegurar el voto universal, libre, secreto, igual y directo ${ }^{1}$, el período pre-electoral es mucho más amplio y da lugar a el respeto del pluralismo político y la existencia de las condiciones necesarias de transparencia y de acceso a la información y a los medios de comunicación para la incoación de la campaña electoral. Por último, el período post-electoral requiere el ejercicio de los derechos políticos, tanto desde el punto de vista de la transparencia del recuento y del

1 Tal y como se deriva del Código de buenas prácticas en materia electoral, Comisión de Venecia, adoptado en 2002, CDL-AD(2002)023rev-spa. 
anuncio de resultados, como desde el punto de vista del contencioso electoral. Las normas que desde el punto de vista del sistema europeo de derechos humanos garantizan los aspectos propios del proceso electoral son muy amplias e implican el uso tanto de normas de hard law como de estándares de soft law.

El Protocolo adicional primero al Convenio Europeo de Derechos Humanos (CEDH), en su artículo 3, enuncia los derechos de participación política que constituyen la base de los derechos que enmarcan el proceso electoral, si bien el sistema europeo ha sido particularmente escueto en su enunciado. El Convenio Europeo es interpretado por el Tribunal Europeo de Derechos Humanos (TEDH), cuyas sentencias, jurídicamente vinculantes, contribuyen a construir los estándares de hard law del derecho electoral. Los derechos ahí enunciados y desarrollados son derechos de dimensión dual, puesto que, aun siendo individuales, tienen una dimensión colectiva y una importancia esencial para la sociedad en su conjunto.

Sin embargo, el patrimonio común electoral no sólo está basado en la jurisprudencia, sino que se ha construido sobre la base de estándares de soft law, tales como recomendaciones, resoluciones, y códigos de buenas prácticas que usan el derecho comparado y señalan cuales son los principios de derecho constitucional compartidos y que dan lugar a un mayor consenso. Este tipo de estándares han sido desarrollados en gran medida por órganos como la Comisión de Venecia, cuyo nombre completo es Comisión para la democracia a través del derecho. Si bien la Comisión es un órgano creado en el seno del Consejo de Europa (COE) en los albores de la caída del muro de Berlín, se trata de un órgano consultivo del COE que, tras sufrir una modificación de sus estatutos en el año 2002, pasó de ser un acuerdo parcial a ser, además, un acuerdo ampliado, lo que le ha permitido acoger en su seno no sólo a todos los Estados europeos miembros del Consejo de Europa, e incluso a alguno no miembro, como Kosovo ${ }^{2}$, sino también a estados de fuera de Europa. En efecto, cuenta con 61 Estados, 14 más que miembros del Consejo de Europa, y tiene a 6 Estados del continente americano como miembros de pleno derecho ${ }^{3}$ y tres como observadores ${ }^{4}$. Esta amplitud de miras es la que, junto con su vasto mandato y sus acuerdos con otras organizaciones internacionales, como la Unión Europea (UE), la Organización para la Seguridad y la Cooperación Europeas (OSCE), o incluso la Organización de Estados Americanos (OEA), ha facilitado el que la Comisión se haya convertido en un catalizador de los estándares constitucionales, propiciando su circulación hacia estándares comunes internacionales ${ }^{5}$.

2 Kosovo se convirtió en miembro de la Comisión de Venecia en 2014, puesto que obtuvo la mayoría de dos tercios necesaria según el Estatuto de la Comisión. Para entrar a formar parte del Consejo de Europa, sin embargo, es necesaria la unanimidad.

3 Brasil, Costa Rica, Chile, Estados Unidos, México y Perú.

4 Argentina, Canada y Uruguay.

5 V. UbedA De Torres, A.: « La régionalisation par la coordination interétatique : le rôle catalyseur de la Commission de Venise », en Doumbe-Bille, S., La régionalisation du droit international, LGDJ, 2012, pp. 147-168. 
La circulación entre los estándares de hard law y los estándares de soft law es un fenómeno patente, ya que ambos contribuyen a reforzarse mutuamente y constituyen la base de la construcción de un derecho internacional electoral global. El objetivo de esta contribución será el de poner de relieve, en una primera parte, los principios claves del derecho electoral, a través del análisis de la jurisprudencia elaborada por el Tribunal Europeo acerca de los derechos de sufragio activo y pasivo. En la segunda parte, se analizará el uso de los estándares de soft law en la construcción del patrimonio electoral común y el papel decisivo que tiene la Comisión de Venecia en la identificación y el desarrollo del mismo. Ejemplos concretos de las interacciones entre la Comisión de Venecia y el TEDH serán puestos de manifiesto, para ilustrar el refuerzo mutuo y el papel complementario que ambos órganos juegan en el entramado institucional, asegurando que los estándares de derecho electoral circulan sobre una base sólida de derecho comparado.

\section{LA PROTECCIÓN DE LOS DERECHOS POLÍTICOS EN EL CONVENIO EUROPEO DE DERECHOS HUMANOS: ANÁLISIS DE LOS PRINCIPIOS EN MATERIA ELECTORAL A LA LUZ DE LA JURISPRUDENCIA}

Huelga decir que la jurisprudencia del Tribunal no ha proporcionado una definición de democracia, aunque ésta se considera el único modelo político compatible con el derecho que emana del Convenio ${ }^{6}$. La democracia representativa y la celebración del proceso electoral se consideran claves en el seno de la jurisprudencia de la jurisdicción europea ${ }^{7}$.

En el seno de esta primera parte, una serie de elementos serán puestos de relieve: en primer lugar, el contenido y las interpretaciones del enunciado de los derechos políticos contenidos en el Convenio; en segundo lugar, se analizarán las particularidades de los elementos interpretativos vertebradores del proceso electoral y de los derechos de participación política; por último, se hará hincapié en los retos recientes y en las conquistas y avances en la protección de los derechos subjetivos del sufragio activo y pasivo, acompañando el análisis de los límites que han sido puestos de manifiesto por la jurisprudencia más reciente.

\section{El alcance del derecho de participación política en el CEDH}

La lectura del Convenio Europeo y de su Protocolo adicional lleva a una primera conclusión ineludible: la consagración de derechos políticos se dirige,

6 Ver en este sentido la sentencia del TEDH Partido comunista unificado c. Turquía párrafo 45.

7 V. sobre la relación del TEDH con el principio democrático, UBEDA DE TorRes, A.: Democracia y derechos humanos en Europa y en América, Reus, Madrid, 2007. 
objetivamente, a la celebración como tal del proceso electoral. Las obligaciones para los Estados que se derivan del artículo 3 del Protocolo I son muy distintas en su enunciado a los demás derechos. En efecto, la disposición parece concentrarse exclusivamente en la obligación de celebrar elecciones y no hace alusión expresa a los derechos individuales de sufragio, si bien la jurisprudencia ha sido clave para matizar y desarrollar dichos derechos.

El peso del factor estatal en la cláusula europea es palpable, dado el enunciado atípico del artículo 3 del Protocolo I, y ha necesitado de mayor desarrollo jurisprudencial. La desaparecida Comisión Europea de Derechos Humanos (Comisión $\mathrm{EDH}$ ), en los primeros momentos, negó incluso la aplicabilidad directa del artículo 3 del Protocolo $\mathrm{I}^{8}$, lo que dio lugar a especulaciones iniciales acerca de si el derecho de voto estaba protegido, si bien fueron disipadas rápidamente, por la Comisión primero, y después por el Tribunal. A pesar de que durante algún tiempo se declararon las demandas relativas a este artículo inadmisibles ${ }^{9}$, la Comisión fue incluyendo poco a poco el derecho de sufragio dentro de la cláusula, declarando a partir de una decisión de 1967 el reconocimiento del sufragio universal ${ }^{10}$. Más adelante, el sufragio pasivo también se incluyó bajo el manto del Convenio, llegando la consagración del cambio jurisprudencial con la célebre (y celebrada) sentencia Mathieu-Mobin y Clerfayt c. Bélgica del TEDH, en la que se pone de manifiesto que «la coloración interestatal» de la redacción del artículo no refleja ninguna diferencia de fondo con las demás disposiciones del Convenio o de sus Protocolos ${ }^{11}$.

Despejada esta primera cuestión acerca del contenido de los derechos individuales, el contenido de las obligaciones estatales relativas a las elecciones también ha ido perfilándose. Así, la referencia del artículo 3 a la elección del cuerpo legislativo ha sido interpretada de forma progresiva, si bien es un límite a la aplicación de la protección del Convenio. En efecto, se han incluido, además de las elecciones al cuerpo legislativo nacional, las elecciones a entidades de tipo federal. Para ello, el criterio a seguir ha sido atender a lo establecido, en el plano interno, por la Constitución y, por tanto, Länder y otras formaciones, así como los Consejos regionales belgas que dan lugar a la demanda en el caso Mathieu-Mobin et Clerfayt, pertenecen a esta categoría ${ }^{12}$. Igualmente están protegidos órganos impor-

8 Así ocurrió en las demandas n. ${ }^{\circ}$ 530/59, contra la RFA, decisión de 4 de enero de 1960 ( $A n$. 3 , p. 184); y en la demanda n. $.^{\circ} 1065 / 61$, c. Bélgica (An. 4, p. 260), en la que la Comisión Europea declara inadmisible la petición de participar en las elecciones organizadas en Bélgica presentada por unos belgas residentes en territorio colonial.

9 García Roca, J., «Del compromiso internacional de los Estados de organizar elecciones libres al derecho de sufragio de los ciudadanos (art. ${ }^{\circ} 3$ P1 CEDH)», García Roca, J. y SANTOlaya, P. (coords.): La Europa de los Derechos. El Convenio Europeo de los Derechos Humanos, CEC, Madrid, 2005, p. 829. Se señala que 710 de los 713 recursos presentados sobre el derecho de voto fueron rechazados hasta 1960.

10 Decisión de inadmisibilidad de la Comisión EDH, Xc. Alemania, de 6 de octubre de 1967, demanda n. ${ }^{\circ} 2728 / 66$.

11 Ubeda De Torres, A. Democracia y derechos humanos en Europa y en América, Reus, 2007, p. 430.

12 Así lo había declarado la Comisión respecto de los Länder austriacos en la decisión de inadmisibilidad X c. Austria, de 12 de Julio de 1976, DR 6, p. 120. Ya discutido durante los trabajos preparatorios a 
tantes dentro de procesos de autodeterminación, aunque la transferencia de competencias de la metrópolis al territorio no haya sido completa ${ }^{13}$. Quedan excluidas aquellas entidades o asambleas sin poder legislativo, puesto que dependen, en última instancia, de un cuerpo legislativo en el sentido del Convenio. La primera parte de la polémica se basó en la petición del derecho de voto al Parlamento Europeo: desde la primera decisión de la Comisión EDH al respecto, en la que entendió, en 1978, que la falta de poderes legislativos del mismo no permitían considerarlo como un cuerpo legislativo y que, por ende, no podía extenderse la protección del derecho de sufragio otorgado por el Protocolo a este órgano ${ }^{14}$, el TEDH, veinte años más tarde, se pronunció en sentido distinto en la famosa sentencia Matthews c. $R U$, considerando al RU responsable de las obligaciones internacionales contraídas en su territorio, aplicables a Gibraltar y que la evolución del Parlamento europeo permitía calificarlo de cuerpo legislativo ${ }^{15}$.

Ello implica que las elecciones municipales y las elecciones presidenciales ${ }^{16}$ no están protegidas, al contrario que lo que ocurre en el ámbito de la Convención Americana de Derechos Humanos. Además, tan sólo las elecciones, y no otros aspectos que podrían encuadrarse dentro de un régimen democrático, derivan de las mismas: las formas de democracia participativa o «semi-directa» han sido excluidas del ámbito del artículo 3 del Protocolo 1 del CEDH por una jurisprudencia constante ${ }^{17}$. La razón de dicha exclusión ha sido el carácter meramente consultivo de los referendos, si bien algunos se utilicen con el fin de derogar una disposición de una ley o en materia de autodeterminación ${ }^{18}$. También se excluyen

petición del RU, en Matbieu-Mobin y Clerfayt se precisa que basta, si el Parlamento está compuesto por dos cámaras, que una de ellas sea elegida conforme al principio de democracia representativa (párrafo 53).

13 Así, se considera como cuerpo legislativo de la Nueva Caledonia a su congreso, sentencia del TEDH Py c. Francia, de 11 de enero de 2005, párrafo 42.

14 Decisión de inadmisibilidad de la Com EDH, Lindsay y otros c. RU, de 8 de marzo de 1979, demanda n. ${ }^{\circ} 8354 / 78$, DR 15 , pp. $247-250-$

15 Sentencia del TEDH Matthews c. RU, de 18 de febrero de 1999, párr. 48.

16 En efecto, la Com EDH lo había declarado así en dos asuntos (Baskauskaite c. Lituania, decisión de 21 de octubre de 1998, demanda no 41090/98, sin publicar, y Habsburg-Lotbringen c. Austria, de 14 de diciembre de 1989 , demanda no $15344 / 89, D . R$. 64, p. 211). Sin embargo, el TEDH ha afirmado que no excluye la posibilidad de aplicar el artículo P1-3 a las elecciones presidenciales, ya que esta disposición debe estar dirigida a regular funciones del cuerpo legislativo. Un jefe de Estado puede tener iniciativa legislativa, o incluso el poder de adoptar leyes y otras vastas prerrogativas en materia legislativa (decisión del TEDH Boskoski c. Ex-República Yugoslava de Macedonia, de 2 de septiembre de 2004, Rec. 2004-VI, párrafo 1). Como, en el caso en cuestión, el Presidente no tenía ninguno de estos poderes, la demanda es declarada inadmisible por incompetencia rationae materiae.

17 Confirmada en la famosa saga relativa a los votos de los presos, puesto que en la decisión Mooban y Gillon c. $R U$, de los demandantes se quejaban de no haber podido votar en el referéndum sobre la independencia de Escocia de 2014 y el TEDH consideró la demanda sobre la base del artículo 3 del Protocolo I inadmisible, decisión de13 de junio de 2017.

18 Así se desprende de la decisión de la Comisión EDH de 10 de julio de 1975, demanda n. ${ }^{\circ}$ 6742/74, D.R. 3, p. 98, si bien los referendos en general, y los de autodeterminación en particular, sí se hallan protegidos en el seno de Naciones Unidas por el artículo 25 del PIDCP (v. observación general del Comité de DH n. ${ }^{\circ} 25$ de 12 de julio de 1996, párrafo 10). 
las elecciones que no sean de carácter político, como las celebradas en el seno de una organización profesional, así como las de tipo administrativo.

\section{Los principios claves de los derechos de participación política en la jurisprudencia}

El Convenio contiene una serie de garantías que aseguran la libertad de las elecciones. Hay dos principios fundamentales, que se han convertido en los pilares sobre los que se ha basado el Tribunal: por un lado, la autenticidad que debe regir todo el proceso electoral; por el otro, la importancia del principio de igualdad, que constituye una guía esencial para evaluar el cumplimiento de las obligaciones estatales.

Respecto del significado de la autenticidad, es un elemento que no aparece expresamente en el Convenio, aunque se discutió mucho y está íntimamente relacionado con el voto libre e igual. De hecho, las elecciones auténticas son aquellas en las que hay una relación directa entre la voluntad de los electores y el resultado de la votación y no hay injerencia en la voluntad de los ciudadanos. La autenticidad se halla, pues, ligada a la idea de libertad de las elecciones, que exige a su vez otros dos requisitos: por un lado, el Estado debe asegurar que la voluntad del elector se forme libremente, lo que entraña una obligación negativa de no intervenir en la misma, y una obligación positiva de asegurar el acceso de los ciudadanos al voto; por el otro, debe reunir las condiciones necesarias para que esa voluntad pueda expresarse también libremente. Ello conlleva la ausencia de un ambiente de represión, ya no sólo en cuanto a las elecciones en sí, sino también respecto de los derechos fundamentales directamente asociados a las mismas, en especial las restricciones a la libertad de expresión y asociación, por su importancia en los procesos electorales.

El principio de igualdad aplicado al proceso electoral se extiende a tres aspectos principales: la existencia de un solo voto por elector, lo que excluye evidentemente votos colectivos o familiares; la igualdad de oportunidades, que puede ser aplicada de forma estricta (que todos los partidos sean tratados de forma exactamente idéntica) o proporcional (en función del número de votos que recibieron), y la igualdad de la «fuerza electoral», que impide el uso de la distribución de circunscripciones electorales para favorecer a determinados partidos.

La jurisprudencia lo ha analizado de forma desigual, ya que el margen de apreciación estatal ha tenido una presencia muy importante. Así, el TEDH ha tenido en cuenta dos elementos claves a la hora de establecer si el artículo 3 del Protocolo adicional I ha sido violado: por un lado, si ha habido un comportamiento arbitrario o que ha tenido un impacto desproporcionado en los derechos individuales y, por el otro, la protección de la libre expresión de la opinión de la sociedad democrática. Así, la igualdad no puede llevar a considerar que tiene que haber una correlación perfecta entre los votos y los representantes políticos, que 
pudiera dar lugar a una preferencia del sistema proporcional frente al mayoritario $^{19}$. De hecho, un sistema que imponga una barrera electoral mínima para participar en las elecciones no ha sido considerado contrario al principio de igualdad ${ }^{20}$, ni siquiera cuando dicha barrera electoral se haya considerado como la más alta de todo el sistema europeo ${ }^{21}$. En fin, cada sistema electoral debe apreciarse a la luz de la evolución política del país, ya que lo que pudiera ser inaceptable en uno puede justificarse en otro ${ }^{22}$.

Finalmente, numerosos aspectos de los procesos electorales también escapan al control jurisdiccional. En materia de financiación de las campañas electorales el sistema europeo concede un amplio margen de apreciación, y se admiten restricciones internas que puedan tener justificación en algún objetivo legítimo y compatible con el principio democrático ${ }^{23}$, aunque ello conlleve como sanción la retirada del derecho a ejercer el cargo del candidato ${ }^{24}$. El sistema establece el marco general en el que se encuadran los procesos electorales, que exige un régimen equilibrado y transparente de financiación o igualdad de acceso a los medios, pero deja a los Estados un amplio margen para la regulación concreta de los mismos. El TEDH ha afirmado que el artículo 3 del Protocolo I no puede ser «un código electoral» con vocación a regular todos los aspectos del proceso electoral ${ }^{25}$.

El desarrollo jurisprudencial del aspecto subjetivo o individual de la participación política ha dado lugar a una construcción de la protección de la democracia a través de los derechos individuales, no exenta de dificultades.

19 Comisión EDH, decisiones Partido liberal y otros c. RU, de 18 de diciembre de 1980, D.R. 21, demanda n. ${ }^{\circ} 8765 / 79$, p. $211 ;$ X c. Islandia, de 8 de diciembre de 1981, D.R. 27 , demanda n. ${ }^{\circ} 8941 / 80$ p. 145 .

20 La Coalición federalista canaria interpuso una demanda ante el Tribunal de Estrasburgo contra España por un asunto relativo al sistema existente, ya que, en las elecciones de la Comunidad Autónoma, y habiendo obtenido más de un $28 \%$ de los votos, no obtuvo ningún escaño en el Parlamento canario. El TEDH consideró que el establecimiento de barreras electorales determinadas para evitar la «atomización» del cuerpo electoral es legítimo, y ello llevó al Tribunal a no admitir la demanda, decisión del TEDH Federación nacionalista canaria c. España, de 7 de junio de 2001.

21 Como fue el caso de la sentencia TEDH, Yumak y Sadakc. Turquía, Gran Sala, párrafos 131-132. A pesar de haber fijado la barrera en el $10 \%$, siendo la más elevada de todos los países, se consideró que no impedía la participación de los candidatos en cuestión.

22 Sentencia del TEDH Mathieu-Mobin y Clerfayt, op. cit., párr. 52.

23 Sentencia del TEDH Bowman c. RU, de 19 de febrero de 1998, donde se planteó la compatibilidad entre el Convenio y una regla de Derecho británico, según la cual los particulares no participantes en la campaña electoral no podían ayudar financieramente a uno de los candidatos. Aunque el Tribunal de Estrasburgo analizó este caso a través de una posible violación del artículo 10, y no del artículo P1-3, consideró que este tipo de restricción, aún en principio inadmisible, podía admitirse en aras de «asegurar la igualdad de los candidatos a las elecciones» (párr. 43). En el caso del Partido Nacionalista vasco-organización regional Iparralde c. Francia, sentencia del TEDH de 7 de septiembre de 2007, el Tribunal estableció que la prohibición de fuentes de ingresos extranjeras a los partidos políticos como tal no era incompatible con el artículo 11 del CEDH, si bien hay salvedades derivadas del derecho de la UE.

24 Como fue el caso en Masson c. Francia, en la que la candidatura del demandante fue anulada por irregularidades en la financiación de la campaña, lo que se consideró compatible con el Convenio Europeo, decisión de inadmisibilidad del TEDH de 14 de septiembre de 1999, párrafo 2.

25 Sentencia del TEDH, Davydov y otros c. Rusia, de 30 de mayo de 2017. 


\section{La protección de los derechos de sufragio activo y pasivo: casos escogidos}

Respecto de las condiciones exigidas para el ejercicio del derecho de sufragio activo, hay una serie de restricciones compatibles con el Convenio Europeo. La primera de ellas, la nacionalidad. El Convenio ha sido restrictivo con el derecho de sufragio de los extranjeros, excluidos de la protección del artículo 3 del Protocolo I, que solo protege las elecciones a cuerpos legislativos, y que no incluye por tanto las elecciones locales. El criterio de residencia también ha dado lugar a una jurisprudencia que ha confirmado la posibilidad, en general, de restringir el derecho de voto por no cumplir con los requisitos de residencia exigidos ${ }^{26}$. En efecto, varias razones, repetidas en numerosos casos, justifican tales restricciones, que se pueden resumir en dos: el especial vínculo entre los candidatos elegidos y los votantes, durante la campaña y durante el mandato, y evitar el fraude electoral ${ }^{27}$. El TEDH ha desarrollado su interpretación basada en el consenso existente entre los distintos países y dando importancia al margen de apreciación, considerando que no existiendo una aproximación común hacia los derechos de voto de los no residentes, son compatibles con el Convenio una serie de situaciones, como la pérdida del derecho de voto tras una serie de años a los nacionales que ya no residen en el país ${ }^{28}$, incluso aunque sean expatriados, no existiendo la obligación de organizar materialmente el voto desde el extranjero ${ }^{29}$. Si tal posibilidad existe, sin embargo, es necesario que el voto sea organizado de forma que se respeten la autenticidad y la libertad de las elecciones ${ }^{30}$. Por último, la edad es el tercer criterio de restricción de las condiciones de ejercicio.

Una de las cuestiones que más dificultades ha suscitado en los últimos tiempos, incluido el posible abandono del sistema del CEDH por parte de algunos Estados por esta razón, ha sido la cuestión del derecho de voto de los presos. Así, frente a la jurisprudencia constante en el pasado, según la cual se podía imponer

26 Decisiones de la Comisión X. y otros c. Bélgica; X. c. RU (11 de diciembre de 1976); X. c. RU (28 de febrero de 1979); X. c. RU (13 de mayo de 1982); decisión de la Comisión Polacco y Garofalo c. Italia (15 de septiembre de 1997).

27 La Comisión EDH los enuncia de la siguiente forma: a) La falta de conocimiento del ciudadano no residente de los problemas cotidianos del país; b) Su imposibilidad de conocer bien los programas electorales y conocer a los candidatos; c) La influencia o adaptación de los programas a los residentes; d) La correlación entre el derecho de voto y el hecho de soportar las consecuencias directas de la elección, Polacco y Garofalo c. Italia, demanda n. ${ }^{\circ}$ 23450/94, decisión de la Comisión EDH de 15 de septiembre de 1997, enunciado también por el TEDH en Hilbe c. Liechtenstein, de 7 de septiembre de 1999.

28 TEDH, Schindler c. $R U$, sentencia de 7 de mayo de 2013, en la que se decidió que el plazo de 15 años para la pérdida del derecho de voto por no residencia no era contrario a las exigencias del Convenio. En la ya mencionada sentencia $P y c$. Francia, el demandante no pudo demostrar los 10 años de residencia exigidos para poder votar en las elecciones al Congreso y el Tribunal consideró que, aunque podría haber casos individuales en el que los vínculos con el lugar pudieran ser constituidos en menos tiempo, el requisito de los diez años de residencia era objetivo para evitar que personas que pasen poco tiempo en Nueva Caledonia, un territorio en fase de transición hacia la adquisición de su plena soberanía, alteren los resultados electorales.

29 TEDH, Sitaropoulos y Giakoumopoulos c. Grecia, Gran Sala, sentencia de 15 de marzo de 2012.

30 TEDH, Riza y otros c. Bulgaria, sentencia de 13 de octubre de 2015. 
la pérdida del derecho de voto a toda persona condenada y mientras estuviese cumpliendo su sentencia ${ }^{31}$, e incluso por un período que excediese al de la condena, el asunto Hirst dio lugar a un giro importante de la cuestión ${ }^{32}$. En efecto, el Sr. Hirst era un condenado por asesinato que se quejó ante el TEDH de que, como pena accesoria a la principal, fue privado de su derecho de voto en las elecciones nacionales y locales del Reino Unido. El TEDH hizo hincapié en que se trataba de una suspensión general y automática de todos los presos convictos, y que, aunque en el caso particular, al ser un delito grave, podría estar justificado, el negar de forma sistemática y automática a todos los presos de sus derechos al voto era incompatible con el artículo 3 del Protocolo I. El Tribunal consideró que toda restricción de derechos fundamentales impuesta de forma automática e indiscriminada, sin que pudiese caber una evaluación caso por caso, era contraria al principio de proporcionalidad. Este caso ha sido seguido por toda una saga de sentencias que siguieron a la negativa del Reino Unido a ejecutar Hirst. El Tribunal abrió además varias vías posibles para facilitar la ejecución y dando plazos para ello. Cinco años después, como no se había avanzado nada, el Tribunal, en Greens y $M T$ c. $R U^{33}$, condenó al Reino Unido como consecuencia de esa falta de ejecución. Al no haber modificado la ley que imponía de manera sistemática la inhabilitación de los presos, y dado el número tan elevado de demandas repetitivas que había recibido, el Tribunal decidió aplicar el procedimiento de las sentencias piloto a este caso y, en virtud del artículo 46 del CEDH, dio al Estado seis meses para hacer la modificación legislativa necesaria para cumplir con lo establecido en Hirst n..$^{\circ}$ 2. Entretanto, todo examen de demandas repetitivas pendientes contra el Reino Unido por este tema fue congelado a la espera de la ejecución de la sentencia, lo que duró hasta 2013, ya, que a. no haber sido aprobada la propuesta legislativa que se hizo, se continuó con el examen de las más de 2000 demandas pendientes ${ }^{34}$. En diciembre de 2017 , el Reino Unido presentó una serie de medidas administrativas de ejecución, que incluía la posibilidad en algunos casos de que los presos pudieran votar, pudiendo salir con este fin con un permiso temporal. Dichas medidas fueron consideradas como apropiadas, y tras la presentación del plan de cumplimento ante el Comité de Ministros, la ejecución fue considerada cerrada en la decisión de diciembre de $2018^{35}$. Un problema más complejo se planteó en la sentencia Anchugov y Gladkov c. Rusia ${ }^{36}$, siempre sobre

31 Comisión EDH, decisión de 6 de octubre de 1967, demanda n. ${ }^{\circ}$ 2728/66, contra Alemania; decisión de la Com EDH de 19 de diciembre de 1974, D.R. 1, p. 87, demanda n. ${ }^{\circ}$ 6573/74; decisión de la Comisión de 4 de julio de 1983, demanda no 9914/82, D.R. 33, p. 244; dec. Com EDH Patrick Holland c. Irlanda, demanda no 24827/94, de 14 de abril de $1998, D . R$. 93-B, p. 15, etc.

32 Sentencia del TEDH Hirst c. RU n. ${ }^{\circ}$, Gran Sala, de 6 de octubre de 2005.

33 Sentencia del TEDH de 23 de noviembre de 2010.

34 Dando lugar a condenas en McHugh y otros c. Reino Unido, de 10 de febrero de 2015 y en Millbank y otros c. Reino Unido, de 30 de junio de 2016.

35 Resolución del Comité de Ministros de 6 de diciembre de 2018, CM/ResDH(2018)467.

36 Sentencia del TEDH del 13 de julio del 2013. 
la prohibición automática e indiscriminada del derecho de voto de los presos, pero que en este caso deriva de un mandato constitucional rígido. Ante la imposibilidad de reformar la Constitución sobre este punto y tras la decisión del Tribunal Constitucional ruso de inejecutabilidad de la sentencia del $\mathrm{TEDH}^{37}$, al menos en lo que a las medidas de carácter general que se derivaban de la misma se refieren, se dio vía libre a Rusia para encontrar formas de interpretar la Constitución de forma compatible con el Convenio. En el plan presentado en junio de 2019 por las autoridades rusas, se explicó que se había modificado el Código penal, para poder hacer cumplir su condena a los convictos por delitos menores en centros sin la consideración de prisiones y, por tanto, dándoles la posibilidad de votar. Con ello, salvaguardaban la Constitución, que prohibía el voto de los presos recluidos por sentencia firme y la hacían compatible con el Convenio y la sentencia del TEDH, puesto que los convictos por delitos de menor gravedad podrían votar. El Comité de Ministros ha declarado el caso cerrado el 25 de septiembre de 2019.

El derecho de sufragio pasivo también se halla sometido a condiciones de edad, nacionalidad y residencia, y, según el TEDH, es compatible con el CEDH que sean aplicadas de forma más estricta que para ejercer el derecho al voto, incluso añadiendo otras condiciones de elegibilidad. El TEDH ha establecido que si bien los Estados tienen la potestad de regular las condiciones de acceso, estas limitaciones deben adecuarse a las bases interpretativas del CEDH: es decir, deben ser conformes con el principio de efectividad de los derechos, que exige derechos efectivos y no ilusorios; deben respetar el principio de proporcionalidad, y, por último, deben salvaguardar la libre expresión del electorado. Así, en el caso de un refugiado ucraniano residente habitualmente en Estados Unidos, que había huido de su país por miedo a represalias políticas y que cumplía los requisitos formales para presentarse a las elecciones, el TEDH decide, teniendo en cuenta todas estas circunstancias, condenar al Estado y exigir la aceptación de su candidatura $^{38}$. Otro ejemplo se produce respecto de los candidatos a las elecciones, y concretamente, acerca del conocimiento del idioma nacional como otro requisito susceptible de restringir el derecho de sufragio pasivo, una cuestión muy vinculada con las minorías y sus derechos políticos. En Podkolzina c. Letonia, la demandante, perteneciente a la minoría rusa, fue sometida a una serie de exámenes específicos para demostrar su dominio del letón, el cual ya había sido acreditado mediante el correspondiente diploma. El Tribunal reconoce que, si bien los conocimientos lingüísticos son un objetivo legítimo para imponer una limitación, la constatación de dichos conocimientos no debe ser arbitraria ni desproporcionada $^{39}$, lo que homogeneiza la jurisprudencia del TEDH con la del Comité de

37 Sentencia del TC de 19 de abril de 2016.

38 Sentencia Melnitchenko c. Ucrania, de 19 de octubre de 2004.

39 Sentencia del TEDH de 9 de abril de 2002, párrafo 35. 
Derechos Humanos de las Naciones Unidas ${ }^{40}$. La situación de las minorías y su participación en los asuntos públicos también ha dado lugar a otra condena en Aziz c. Chipre, donde debido a la situación en la isla, los miembros de la comunidad turco-chipriota residentes en la zona controlada por el gobierno de Chipre no podían inscribirse en el censo y ejercer su derecho al voto, mientras que la comunidad greco-chipriota $\mathbf{s i ́}^{41}$.

En fin, la jurisprudencia del TEDH ha sido esencial para definir los contornos de los derechos de participación política, pero la interacción con la Comisión de Venecia ha permitido desarrollar aún más los principios del derecho electoral, contribuyendo a la creación de un acervo jurídico común en este campo.

\section{LA PROTECCIÓN DE LA DEMOCRACIA A TRAVÉS DE LOS ESTÁNDARES DE SOFT LAW: LA CONTRIBUCIÓN DE LA COMISIÓN DE VENECIA}

La Comisión de Venecia ha contribuido de forma significativa al desarrollo de estándares internacionales en materia electoral, actuando como un auténtico catalizador del consenso estatal hacia un consenso regional y universal. Sus rasgos distintivos y su trabajo como órgano consultivo, de gran influencia en la construcción de un derecho constitucional común en el ámbito electoral, serán objeto de análisis en primer lugar. A continuación, se señalan los principios claves y los estándares desarrollados por la Comisión, a través de mecanismos tales como el Código de buenas prácticas en materia electoral. Por último, el refuerzo de dichos estándares internacionales en materia electoral a través de las interacciones entre la jurisprudencia de los tribunales internacionales de derechos humanos, y sobre todo del TEDH y la Comisión de Venecia serán puestos de relieve a través de ejemplos concretos.

\section{La Comisión de Venecia y su papel determinante en la identificación de estándares internacionales en materia electoral}

La principal tarea de la Comisión de Venecia es ayudar a los Estados a identificar valores comunes compartidos, basados en los tres pilares del Consejo de Europa: democracia, estado de derecho y derechos humanos. La Comisión emite opiniones, en las que comparte sus puntos de vista sobre enmiendas constitucionales concretas y legislación clave de un Estado en particular cuya situación le

40 En efecto, en Ignatane c. Letonia, comunicación n. ${ }^{\circ} 884 / 1999$, de 25 de julio de 2001, en el que se discute también acerca de la negativa de inscribir en las listas de candidatos a una ciudadana letona de origen ruso, el Comité se pronuncia en términos muy parecidos.

41 Sentencia del TEDH de 22 de junio de 2004. 
haya sido sometida. En sus opiniones, que no son vinculantes, la Comisión ofrece críticas, cuando sea necesario, de cualquier disposición nueva que no se ajuste a las normas establecidas, y también recomienda e identifica nuevos estándares. Los Estados que han sido el objeto de dicha opinión sobre las enmiendas constitucionales o legislativas en cuestión, tienen la opción de seguir o no estas recomendaciones y adaptarlas a su contexto nacional específico. No existe una solución común que funcione para todos los países y que pueda ser recomendada en todas sus opiniones, lo que hace que la tarea de la Comisión de Venecia para establecer los estándares y, al mismo tiempo, hacer recomendaciones válidas a nivel nacional sea difícil y exija un conocimiento profundo del contexto especifico nacional.

La Comisión de Venecia debe cooperar con los Estados, lo que es más fácil de lograr de manera efectiva cuando son los Estados los que han solicitado explícitamente esta cooperación, pero sin perder su neutralidad, demostrando que es una entidad técnica jurídica y no política. Las opiniones de la Comisión deben ser percibidas como imparciales e independientes para que tengan éxito y no se menoscabe su autoridad. Para lograr su complejo objetivo de identificar estándares constitucionales comunes, la Comisión no sigue un modelo de supervisión vertical, sino de cooperación estatal. También contribuye a la transformación de los ordenamientos jurídicos nacionales al ayudarlos a adaptarse de manera preventiva a las normas internacionales y regionales. La Comisión de Venecia internacionaliza, pues, los ordenamientos jurídicos nacionales mediante la identificación de elementos comunes y la creación de puentes entre losestándares de soft law y los estándares existentes de hard law.

Las características clave que explican cómo la Comisión identifica estándares tienen que ver principalmente con su metodología flexible. En lo que respecta a quién puede realizar una solicitud a la Comisión de Venecia, hay tres tipos posibles de «clientes». El artículo 3.2 del Estatuto de la Comisión de Venecia establece que: «La Comisión puede dar, dentro de su mandato, opiniones bajo petición presentada por el Comité de Ministros, la Asamblea Parlamentaria, el Congreso de Autoridades Locales y Regionales de Europa, la Secretaría General o por un estado u organización internacional, o cuerpo participante en el trabajo de la Comisión. Cuando un estado pida una opinión de un asunto concerniente a otro estado, la Comisión debe informar al estado concerniente y, a menos que los dos estados lo acuerden, debe presentar el asunto al Comité de Ministros.» La Comisión de Venecia, por lo tanto, busca principalmente estar a disposición de los Estados miembros, que incluso pueden realizar una solicitud interestatal relativa a otro Estado miembro, aunque el procedimiento no ha tenido mucho éxito. Además, el Estatuto fue revisado a este respecto para exigir que la solicitud interestatal sea precedida por el acuerdo previo de los dos Estados interesados o si no, que sea enviada por el Comité de Ministros ${ }^{42}$. Los Estados no miembros también

42 La práctica ha sido compleja. Rumanía lo pidió en 2001 respecto la legislación húngara sobre minorías, lo que se resolvió con la preparación del estudio general en 2001 sobre el trato preferente de las minorías 
pueden pedir opiniones a la Comisión, pero no directamente, sino a través de alguna organización que coopere con ella ${ }^{43}$.

Dentro de cada Estado, la solicitud puede provenir tanto del gobierno, como del Parlamento (a través de su presidente o de un acuerdo de los partidos que se hallen representados) e incluso, en ciertos casos, de la Oficina del Defensor del Pueblo. Sin embargo, para evitar que la Comisión se utilice como una forma de obstruir reformas internas o como instrumento de la oposición, los partidos políticos no pueden enviar solicitudes de forma independiente sin el apoyo de una institución oficial, ya sea el Parlamento o el gobierno, ni las autoridades locales sin el consentimiento del Estado central.

El segundo grupo de actores que pueden desencadenar el trabajo de la Comisión de Venecia son los órganos principales del Consejo de Europa: el Comité de Ministros, la Asamblea Parlamentaria (PACE), el Congreso de Autoridades Locales y Regionales y el Secretario General. Las solicitudes provenientes de estos órganos ayudan a institucionalizar el trabajo de la Comisión de Venecia y a consolidarlo como un actor que no depende totalmente de la voluntad de los Estados para ejercer su trabajo. En los últimos años, estos mecanismos institucionales se han reforzado. En el caso de la Asamblea Parlamentaria, ha desarrollado un papel cada vez más importante en la incoación de opiniones. También ha usado sus poderes para abordar situaciones problemáticas, como por ejemplo en relación con el uso de decretos de emergencia y de legislación especial en Francia ${ }^{44}$ y Turquía $^{45}$. El Secretario General también ha desempeñado un papel clave, solicitando el primer análisis sobre la compatibilidad de ciertas «acciones» (no legislativas) tomadas por el gobierno y el Parlamento de Rumania con el Estado de Derecho ${ }^{46}$, así como del referéndum en Crimea ${ }^{47}$. Este último ejemplo es crucial en el campo electoral. Muestra claramente cómo el uso de normas de soft law, como el Código de buenas prácticas en materia de referendos, y el refuerzo mutuo de éstas con normas vinculantes, como la Constitución nacional, llevaron a la Comisión de Venecia a concluir que el referéndum se había celebrado en clara violación de las normas europeas.

Por último, cualquier organización internacional que participe en el trabajo de la Comisión puede pedir opiniones, siempre que hayan sido invitadas a participar una vez en la sesión plenaria. Los actores más relevantes, sobre todo en el ámbito electoral, han sido la OSCE/ODIHR y la Unión Europea. En el campo de las elecciones, la OSCE/ODIHR ha tenido un impacto clave, ya que existe un acuerdo en

nacionales por el Estado afín. Azerbaiyán también lo utilizó respecto del preámbulo de la Constitución de Armenia acerca de la reunificación de la región del Nagorno-Karabaj, que fue abandonada ulteriormente.

43 Como ha sido el caso de Bolivia.

44 Opinión de 2016, CDL-AD(2016)006.

45 Opiniones de 2017, CDL-AD(2017)007 y CDL-AD(2017)021.

46 Opinión de 2012, CDL-AD(2012)026.

47 Opinión de 2014, CDL-AD(2014)002. 
virtud del cual cualquier solicitud de opinión de la ODIHR o de la Comisión de Venecia debe tratarse como un proyecto conjunto. En la práctica, por lo tanto, casi todas las opiniones electorales, incluidas las relacionadas con los partidos políticos y la financiación de campañas electorales, han sido opiniones conjuntas. La OSCE no solo trae a la Comisión de Venecia un grupo de Estados más amplio y diferente, sino también una experiencia práctica en el campo. Los informes electorales elaborados por la ODIHR durante la observación de las misiones electorales son una fuente de información muy importante utilizada como base para el análisis realizado en las opiniones y recomendaciones de la Comisión. Una vez que la opinión ya está siendo elaborada, el Consejo para las Elecciones Democráticas completa el mecanismo institucional. Es un organismo tripartito, compuesto por miembros de la Comisión de Venecia, la Asamblea Parlamentaria y el Congreso de Autoridades Locales y Regionales. El Consejo discute cualquier opinión relacionada con el campo electoral antes del debate en el plenario, asegurando que se tome un enfoque coherente, especialmente en lo que respecta al uso de las fuentes.

En materia electoral, la Comisión puede proporcionar además un apoyo técnico que va más allá de asesorar la legislación. Ucrania ha sido un ejemplo paradigmático de este tipo de cooperación, ya que se han establecido contactos con un grupo de expertos en derecho electoral en el país con el que ha trabajado en muchos proyectos diferentes después del Maidan ${ }^{48}$, que han resultado en un intercambio beneficioso de experiencias e ideas que pueden ayudar a los expertos nacionales en su tarea de redacción. No solo ha ocurrido en este caso, sino en muchos otros, incluyendo Armenia o Georgia. La Asamblea Constituyente de Túnez del país consultó a la Comisión en varias ocasiones después de 2012 durante la redacción de la Constitución, incluso sobre la cuestión de la elección del tipo de sistema democrático para el país y el sistema electoral. El gobierno tunecino terminó solicitando una opinión formal a la Comisión sobre el texto constitucional antes de su votación final ${ }^{49}$.

La influencia que ha tenido la Comisión en la identificación de estándares en materia electoral se ha visto reforzada por el desarrollo de los mismos en sus opiniones.

\section{La Comisión de Venecia y su influencia en el desarrollo de estándares electorales internacionales}

La Comisión ha desarrollado fuentes de soft law y contribuido a la identificación de estándares de hard law en el campo electoral. En lo que respecta a las

48 Los acontecimientos que llevan el nombre de la famosa plaza de Kiev llevaron a la salida del ex-presidente Yanukovich en 2014.

49 Opinión de 2013, CDL-AD(2013)032. 
normas de soft law, la Comisión utiliza, cuando estén disponibles, los informes y resoluciones de la PACE y del Comité de Ministros del Consejo de Europa. El uso de las resoluciones de este último parece particularmente apropiado ya que el Comité de Ministros es el órgano político clave del Consejo. Con respecto a la PACE, la interacción es bastante intensa debido a la cooperación reforzada e institucionalizada.

Primero, desde 2004, la Comisión de Venecia tiene un acuerdo con la PACE para acompañarla en sus misiones electorales ad hoc de observación en los Estados miembros. La Asamblea invita a la Comisión de manera sistemática cuando ésta ha emitido previamente una opinión sobre la legislación electoral del Estado en cuestión. El papel de la Comisión de Venecia durante estas misiones consiste en brindar asistencia técnica legal durante la observación, explicando los aspectos jurídicos relevantes de la legislación electoral nacional vigente y recordando a los miembros de la Asamblea que componen la delegación aquellos elementos problemáticos desde el punto de vista de los estándares. Al final de cada misión, la Asamblea Parlamentaria publica un informe detallado sobre los resultados de la observación de las elecciones en ese país específico, que a menudo se coordina con las otras organizaciones especializadas presentes, como la OSCE/ODIHR, la Asamblea Parlamentaria de la OSCE y, a veces, el Parlamento Europeo de la UE. Este informe final se prepara con la cooperación de la Comisión de Venecia, que incluye el análisis del marco legal. Los informes de la PACE se utilizan luego como una fuente de información para preparar opiniones y conocer el contexto nacional. La cooperación con el Congreso sigue el mismo mecanismo, aunque no haya, como con la PACE, un acuerdo formal específico.

En segundo lugar, la cooperación con la PACE también lleva a desarrollar nuevos estándares con la preparación de estudios de derecho comparado. El objetivo es identificar tendencias e incluso enriquecer el debate conjunto. Por ejemplo, la PACE invitó a la Comisión de Venecia a elaborar un informe sobre los métodos de nombramiento de candidatos dentro de los partidos políticos ${ }^{50}$, que fue luego ampliamente utilizado por la Asamblea ${ }^{51}$. El Congreso también ha invitado a la Comisión de Venecia a comentar e informar sobre temas clave, como sobre la situación de los votantes que residen de facto en el extranjero ${ }^{52}$. Precisamente sobre la base de las fuentes utilizadas, de su experiencia práctica en los países, y también de estos estudios generales, la Comisión ha desarrollado varios documentos que establecen estándares clave en el campo electoral. El más importante es, sin lugar a dudas, el Código de Buenas Prácticas en materia electoral, adoptado en

50 Informe de 2015, CDL-AD(2015)020.

51 Asamblea Parlamentaria, resolución 2111, 2016, sobre la mejora de la participación de las mujeres en la representación política.

52 Comisión de Venecia, Informe sobre los votantes residentes en el extranjero, CDL-AD(2015)040; Congreso de Autoridades Locales y Regionales, Listas electorales y votantes que residen de facto en el extranjero, recomendación 369 y resolución 378, de 2015. 
$2002^{53}$. Aunque no es vinculante, se ha convertido en un texto de referencia que ha ayudado a dar coherencia y construir un ius commune en el campo electoral. El Código ha permitido a la Comisión ser más sistemática en su enfoque e identificar más eficazmente el consenso que resulta del derecho comparado. Sin embargo, también requiere precaución a la hora de identificar estándares en el campo electoral: primero, no todos los aspectos analizados en las opiniones pueden dar lugar a recomendaciones. Por ejemplo, la elección del sistema electoral es una cuestión demasiado vinculada a la soberanía estatal y la Comisión de Venecia no se ha pronunciado sobre la bondad o los problemas de algunos sistemas frente a otros. Tan solo en algunas ocasiones ha hecho una excepción y ha criticado cambios de sistemas, justificando su crítica a través en dos aspectos claves: uno, el de la falta de estabilidad del derecho electoral, dado que los cambios repentinos previos a las elecciones no fomentan la confianza en el sistema; el segundo, la experiencia práctica deriva de los informes emitidos por otras organizaciones expertas que revelaban muy claramente los problemas de tal sistema electoral en el contexto del país en cuestión. En esas ocasiones, la Comisión ha tomado postura en sus opiniones desaconsejando ciertos sistemas electorales, siempre contextualizando mucho sus críticas $^{54}$.

Además, el Código de buenas prácticas en materia electoral distingue entre los principios electorales (sufragio igual, universal, libre, secreto y directo) y ciertos elementos necesarios para su implementación: el respeto de los derechos fundamentales, las garantías procesales y la estabilidad electoral. La lucha contra la corrupción como tal o la idea de la responsabilidad electoral no se incluyen explícitamente entre estos elementos clave. El Código contiene una selección de principios de minimis, completada por algunas otras recomendaciones basadas en buenas prácticas, pero no es un conjunto de estándares rígidos y ha sido modificado. Se han adoptado varias declaraciones interpretativas a este respecto a la luz de los problemas recurrentes existentes en algunos países, por ejemplo, la declaración interpretativa de 2016 sobre la publicación de las lista de votantes ${ }^{55}$. El elemento del voto secreto, la confidencialidad, responde a una finalidad básica esencial: evitar las presiones en el momento de emitir el voto, incluyendo la abstención. Ello implica que no sólo se impone una obligación en ese sentido al Estado

53 Comisión de Venecia, CDL-AD(2002)023rev2-cor-e.

54 Así, en el caso de Ucrania, tres sistemas electorales distintos han sido utilizados durante los últimos 18 años: un sistema puramente proporcional de listas cerradas, uno mayoritario con circunscripciones únicas y uno mixto, en el que la mitad de los diputados son elegidos en circunscripciones uninominales y la otra mitad de forma proporcional en una circunscripción única. El sistema mixto se reintrodujo en 2011 y fue criticado sobre la base de los informes de observación electoral de la OSCE y de otros actores internacionales, señalando que si bien es la elección soberana del Estado, los procesos electorales con sistema mixto y mayoritario uninominal habían dado lugar a problemas muy concretos en Ucrania en elecciones pasadas, que deberían ser tenidos en cuenta a la hora de volver a usarlo (Opinión de 2011, CDL-AD(2011)037, para. 22).

55 Declaración interpretativa del Código de buenas prácticas en materia electoral relativa a la publicación de la lista de votantes que han participado en las elecciones, CDL-AD(2016)028. 
a la hora de votar, sino que también supone una obligación para el votante, cuyo voto debe permanecer anónimo.

\section{Las interacciones entre la Comisión y el TEDH en materia electoral}

Finalmente, la Comisión de Venecia no solo emite sus opiniones basadas en normas de soft law. El uso sistemático de la jurisprudencia del Tribunal cuando es pertinente en sus opiniones y las influencias mutuas entre el TEDH y la Comisión de Venecia, tanto oficiales como oficiosas, han fomentado la construcción conjunta y el refuerzo de los estándares jurídicos electorales existentes.

La primera instancia en la que los dos órganos han desarrollado influencias mutuas se produce cuando es el Tribunal el que invita a la Comisión de Venecia a intervenir como amicus curiae en un asunto pendiente. En el campo electoral, la primera intervención fue sobre el tema de la prohibición de contribuciones financieras a partidos políticos de fuentes extranjeras, lo que dio lugar a una extensa opinión sobre la Probibición de las contribuciones financieras derivadas de fuentes externas a los partidos políticos, adoptada en marzo de $2006^{56}$. Dicha opinión fue muy utilizada por el Tribunal, que estableció que la prohibición de dichas fuentes de ingresos extranjeras como tal no era incompatible con el artículo 11 del CEDH, ya que la propia Comisión de Venecia había demostrado la falta de consenso entre los Estados europeos en la materia (caso del Partido Nacionalista vasco-organización regional Iparralde c. Francia ${ }^{57}$ ).

Tras esta invitación inicial, ha sido la propia Comisión la que ha solicitado intervenir ante el Tribunal en varias ocasiones claves: una de ellas, es la del célebre caso Sejdić y Finciv. Bosnia y Herzegovina ${ }^{58}$, relativo a la violación del principio de no discriminación basada en el origen étnico en el acceso a cargos públicos ${ }^{59}$. De esta manera, la interacción entre la Corte y la Comisión de Venecia llevó a una mayor coherencia y sincronicidad entre los dos en el uso de las fuentes. Además, la Comisión de Venecia utiliza regularmente el CEDH y la jurisprudencia del Tribunal Europeo, dando así un efecto horizontal a la Convención, ayudando a

56 CDL-AD(2006)014.

57 TEDH, sentencia de 7 de septiembre de 2007. Es interesante señalar que aunque la sentencia no contradice lo establecido por la Comisión de Venecia, tampoco abraza abiertamente la necesidad de prohibición de fuentes extranjeras, sino que hace que el Tribunal considere que se trata de una cuestión que entra dentro del margen de apreciación estatal.

58 TEDH, sentencia de Gran Sala de 22 de diciembre de 2009.

59 El informe de la Comisión de Venecia se adoptó en octubre de 2008, CDL-AD(2008)027. En esta sentencia, el Tribunal no sólo usó la tercera intervención preparada por la Comisión, sino también otras opiniones previas, como la emitida sobre la situación constitucional en Bosnia y los poderes del Alto Representante (CDL-AD(2005)004, de 11 de marzo de 2005), la relativa a las diferentes propuestas para las elecciones presidenciales (CDL-AD(2006)004, de 20 de marzo de 2006) y la opinión sobre la reforma constitucional de 2006 (CDL-AD(2006)019 de 12 de junio de 2006. 
difundir su impacto más allá de un caso específico (a través del llamado efecto interpretativo).

Rywin contra Polonia ${ }^{60}$ también ha dado lugar a un diálogo directo entre el Tribunal de Estrasburgo y la Comisión en un asunto relativo a las garantías y los poderes de las comisiones parlamentarias de investigación y su relación con los procesos judiciales. El caso tenía como telón de fondo a un conocido productor de cine, pero también a muchas autoridades, incluyendo al Primer Ministro polaco, envueltos en un escándalo de corrupción durante el proceso parlamentario de adopción de la ley de radiodifusión pública. La Comisión de Venecia expuso en su informe que, si bien los procedimientos seguidos ante las comisiones parlamentarias no están protegidos por las garantías del artículo 6 del Convenio, y su carácter público no es ilegítimo, las injerencias en los posibles procesos judiciales posteriores deben ser evitadas y tratadas con la máxima cautela. Sin embargo, el descubrimiento de indicios penales por una comisión parlamentaria de investigación no debería como tal paralizar la misma, sino que ésta debería informar de dichas cuestiones a la fiscalía y seguir su curso, siendo los procedimientos judiciales y parlamentarios separados e independientes entre $\mathrm{si}^{61}$. El Tribunal Europeo tomó una decisión muy ajustada (4 votos contra tres), considerando que no había habido en este caso una violación del principio de presunción de inocencia y, por tanto, violación del Convenio.

El asunto Berlusconi c. Italia ha sido un ejemplo reciente de esta saga judicial de intercambios entre la Comisión y el TEDH, en el que se planteaban las garantías necesarias que deben acompañar a una inhabilitación y consecuente revocación de un parlamentario, en este caso el ex-Presidente Berlusconi. Este se había quejado de que, en virtud de la entrada en vigor en 2012 de la ley 190/2012, el gobierno Monti había establecido por decreto las condiciones de inhabilitación de todo diputado y senador, quedando la decisión final de invalidación de candidaturas y de inhabilitación derivada de una pena principal en manos del Parlamento. Habiendo sido condenado por fraude fiscal en 2012 a cuatro años de cárcel, y a una pena accesoria de cinco años de inhabilitación, que se redujeron después a dos años, el Senado italiano decidió invalidar la elección del señor Berlusconi como senador en febrero de 2013 y le inhabilitó para ejercer su mandato público. La Comisión recordó que toda limitación debe ser restrictiva y en ningún caso general, indiferenciada y desproporcionada, pero las limitaciones son posibles. Cuando la perdida de dicho mandato no es inmediata, sino que se deriva de la decisión final del parlamento, las garantías procesales implican la posibilidad para el parlamentario o senador de presentar los argumentos que considere pertinentes ante el Parlamento, tener una audiencia pública y derecho a abogado, así como a la publicidad de su caso ${ }^{62}$. El asunto ha sido archivado a la petición del

60 TEDH, sentencia de 18 de febrero de 2016.

61 Comisión de Venecia, CDL-AD(2014)013, amicus curiae adoptado en marzo de 2014.

$62 \operatorname{CDL}(2017) 029$. 
propio señor Berlusconi ante el TEDH, al considerar que la inhabilitación ya no existe y que, por tanto, la demanda carecía de sentido ${ }^{63}$.

En Mugemangango c. Bélgica, el Tribunal pidió a la Comisión un informe amicus curiae sobre las garantías procesales necesarias que deben existir en la contestación de resultados electorales y la denuncia de irregularidades en la repartición de escaños. Recordando el Código de buenas prácticas, la Comisión reiteró la necesidad de que cualquier procedimiento de contestación implique para los candidatos el derecho de poder llevar sus demandas ante un órgano independiente e imparcial, suficientemente distinto del ejecutivo y del legislativo, en el que pueda tener una audiencia pública y aportar las pruebas que considere oportunas $^{64}$. La Gran Sala del TEDH se ha pronunciado en julio de 2020, condenando a Bélgica de forma unánime por el procedimiento establecido tras la contestación de los resultados electorales, al considerar que el Parlamento balón no reunía los requisitos de imparcialidad necesarios para haber asegurado un examen efectivo de las demandas interpuestas ${ }^{65}$.

Otro segundo punto de encuentro se produce cuando la Comisión proporciona orientación y asesoramiento sobre cómo el Estado puede aplicar mejor las sentencias del Tribunal u ofrece soluciones para superar posibles obstáculos de ejecución. Rusia ha protagonizado el episodio más reciente, tras la adopción del cambio de la legislación del Tribunal Constitucional en diciembre de 2015, para dar a dicho órgano la posibilidad de valorar la posible imposibilidad de ejecución de las sentencias de Estrasburgo. Tras el cambio legislativo, en febrero de 2016, el Ministro de justicia ruso pidió al Tribunal Constitucional sobre la base de la nueva legislación que se pronunciase acerca de la imposibilidad de cumplimiento de varias sentencias, entre ellas la sentencia ya estudiada Anchugov y Gladkov (vid supra sección I.3).

En fin, el último punto de encuentro, quizás el más importante, es el que se deriva del uso de la jurisprudencia del TEDH en las opiniones, y su adaptación en el desarrollo de recomendaciones específicas para los Estados cuyas legislaciones son objeto de análisis, así como el refuerzo de los estándares a través de la jurisprudencia a través de los estudios generales ya mencionados. Como ejemplos recientes cabe señalar el informe en materia de mandato revocatorio de alcaldes y representantes locales ${ }^{66}$ o los informes sobre los límites de los mandatos presidenciales y parlamentarios y locales, incluyendo las cuestiones de la relección de presidentes ${ }^{67}$.

63 Decisión de la Gran Sala del TEDH de 27 de noviembre de 2018.

64 Comisión de Venecia, CDL-AD(2019)021, amicus curiae adoptado en octubre de 2019.

65 TEDH, sentencia de Gran Sala de 10 de julio de 2020.

66 Comisión de Venecia, CDL-AD(2019)011 rev, Informe sobre el mandato revocatorio de alcaldes y representantes locales, adoptado en junio de 2019.

67 Comisión de Venecia, CDL-AD(2018)010, Informe sobre los límites a la reelección Parte I — Presidentes, adoptado en marzo de 2018; CDL-AD(2019)007, Informe sobre los límites a la reelección Pate II, Miembros del Parlamentoy Parte III Representatives y autoridades administrativas regionales y locales, adoptado en marzo de 2019. 


\section{REFLEXIONES FINALES}

La identificación de estándares universales en el campo electoral puede ser más complejo que en materia de la protección de los derechos humanos o del Estado de derecho, especialmente si dichos estándares no sólo conciernen el llamado «derecho humano a la democracia», directamente relacionado con el ejercicio de los derechos de sufragio activo y pasivo, sino que incluyen la naturaleza misma del sistema electoral. Además de las afirmaciones muy contextualizadas acerca de la adecuación posible de un determinado sistema electoral sobre la base de una práctica concreta, a las que se ha hecho referencia previamente y que es un aspecto que no puede ser extrapolable o «universalizable», hay otros muchos elementos desarrollados por el Tribunal en su jurisprudencia y que aparecen reflejados en las opiniones y estudios de la Comisión de Venecia. Así, en el mencionado estudio sobre los mandatos presidenciales, se examinan las bases de un posible «derecho humano a la reelección», para concluir que, si bien hay una relación entre el derecho al sufragio pasivo y la reelección, ésta es un elemento de funcionamiento institucional y no un derecho como tal, lo cual no implica que las limitaciones establecidas no deban perseguir un objetivo legítimo, ser razonables y necesarias en toda sociedad democrática ${ }^{68}$, aplicando pues a jurisprudencia bien establecida por el TEDH a toda restricción de derechos.

Otro reto es el de considerar que las fuentes en una materia tan cercana a la soberanía del Estado, como es la electoral, se halla en manos de instituciones internacionales que no son de representación política, y por tanto son criticadas por su falta de legitimidad democrática. Para contrarrestar tales críticas, hay dos elementos que pueden ser mencionados de forma preferente: en lo que respecta a la jurisprudencia del TEDH, puede decirse que ha sido cautelosa en su respeto de la particularidad estatal y del margen de apreciación de los Estados. En cuanto a la Comisión de Venecia, ha desarrollado una estructura específica para las opiniones, particularmente las emitidas en el campo electoral, que ayuda a la sistematización de estándares y a una aproximación coherente. Además, todas las opiniones sobre reformas electorales siguen siempre una estructura común e incluyen un análisis de la estabilidad de la ley electoral y el proceso que lleva a su adopción; la elección del sistema electoral; la lista de votantes registrados; cómo es la administración electoral; el uso de los recursos administrativos; los aspectos de la campaña y la financiación electoral; equidad y libertad en el acceso a los medios de comunicación; la representación de mujeres y, finalmente, el contencioso electoral.

La jurisprudencia del TEDH, vinculante para los Estados hacia los que se dirige, no puede por sí sola cubrir todos los ámbitos del derecho electoral. Las interacciones con la Comisión y el uso del Código de buenas prácticas en materia electoral de la Comisión de Venecia y de la fórmula de amicus curiae han sido

68 Ibidem. 
claves han jugado un papel relevante en el desarrollo de estándares y de un acervo jurídico común, contribuyendo no solo al cumplimiento, sino a una construcción sólida y coherente de un patrimonio electoral común basado en el derecho comparado.

\section{$* * *$}

TiTLE: Electoral standards in the case-law of the European Court of Human Rights and the Venice Commission code of good practice on electoral matters

ABSTRACT: The identification and consolidation of human rights standards is part of the work of the European Court of Human Rights. However, the applicability of the European Court's case-law to the construction of common standards in the field of democracy and elections is one of the most difficult areas, as electoral law is generally part of the sovereign choice of the State. The Court has developed a rich case-law on the rights to political participation on the basis of Article 3 to Protocol I. In spite of the particular content of this Article, different of the other rights recognised in the European Convention on Human Rights, there has been a clear evolution towards a protection of both the individual and the dual nature of political rights. This development would have been incomplete without the work and the interactions with the Council of Europe's Venice Commission, which full name is "Commission for Democracy through Law», which largely contributes to the development of standard-setting processes, acting as a catalyser of the States' will to reach a European consensus. The Venice Commission is an international body that has a diplomatic dimension: it must cooperate with States, and, at the same time, it must issue opinions and recommendations that are not always easy to implement (and not always followed) at the national level. This paper focuses on the complex role played by the European Court of Human Rights and the Venice Commission in successfully setting common European standards and good practices in the field of elections. This article explores first the hard law standards as developed by the European Court of Human Rights in its case-law in the field of elections. It further examines the role of the Venice Commission and its working methods when identifying soft law standards in the electoral field and how, through its interactions with the Court, reinforces these standards and hard law standards to promote an electoral ius commune.

RESUMEN: La identificación y consolidación de los estándares de derechos bumanos forma parte de la labor del Tribunal Europeo de Derechos Humanos. Sin embargo, el impacto de la jurisprudencia del Tribunal Europeo en la construcción de normas comunes en el ámbito de la democracia y de las elecciones es una de las cuestiones más complejas, ya que el derecho electoral es generalmente parte del ámbito de soberanía estatal. El Tribunal ha desarrollado una rica jurisprudencia sobre los derechos a la participación política a partir del artículo 3 del Protocolo I. A pesar del enunciado particular de este artículo, diferente de los demás derechos reconocidos en el Convenio Europeo de Derechos Humanos, ha habido una clara evolución hacia una protección naturaleza tanto individual como dual de los derechos políticos. Este desarrollo habría sido incompleto sin el trabajo y las interacciones con la Comisión de Venecia del Consejo de Europa, cuyo nombre completo es «Comisión para la Democracia a través del Derecho", que contribuye en gran medida al desarrollo de procesos normativos, actuando como catalizador de la voluntad de los Estados para alcanzar un consenso europeo. La Comisión de Venecia es un organismo internacional que tiene una dimensión diplomática: debe cooperar con los Estados y, al mismo tiempo, debe emitir dictámenes y recomendaciones que no siempre sean fáciles de aplicar (y que no siempre se siguen) a nivel nacional. Este trabajo se centra en el complejo papel desempeñado por el Tribunal Europeo de Derechos Humanos y la Comisión de Venecia para promover con éxito el establecimiento de normas europeas comunes y buenas prácticas en el ámbito electoral. La contribución explora primero las normas de hard law desarrolladas por el Tribunal Europeo de Derechos Humanos en su jurisprudencia en el ámbito de las elecciones. Examina además el papel de la Comisión de Venecia y sus métodos de trabajo a la hora de identificar los estándares de soft law en el campo electoral y cómo, a través de sus interacciones con la Corte, refuerza dichos estándares y el hard law para promover un ius commune electoral. 
KeY wORDS: European Court of Human Rights, European Convention on Human Rights, elections, political rights, Venice Commission, Code of good practice in the electoral field.

Palabras Clave: Tribunal Europeo de Derechos Humanos, Convenio Europeo de Derechos Humanos, elecciones, derechos politicos, Comisión de Venecia, Código de buenas prácticas en materia electoral.

FECHA DE RECEPCIÓN: 10.02.2020 FECHA DE ACEPTACIÓN: 09.09.2020 